Licenciado en Historia por la Facultad de Filosofía y Letras de la Universidad Autónoma de Chihuahua. Máster en Educación Superior por la misma institución. Director del sistema Radio Universidad de la Universidad Autónoma de Chihuahua y presidente de la Red de Radios Universitarias de México (RRUM). Actualmente doctorante en el Instituto de Pedagogía Crítica.

Historial editorial Recepción: 10 de septiembre de 2020. Revisión: 14 de octubrre de 2020. Aceptación: 23 de noviembre de 2020. Publicación: 13 de diciembre de 2020. 


\title{
Las Fake news, enemigas del periodismo, la comunicación y los Derechos Humanos
}

\author{
Fake news, enemies of journalism, \\ communication and Human Rights
}

\section{As fake news, inimigas do jornalismo, da comunicação e dos direitos humanos}

\author{
Marco Gutiérrez Mendoza \\ Instituto de Pedagogía Critica \\ marcocchib@gmail.com
}

\section{Resumen}

Una de las labores sustanciales del periodismo y la comunicación es la de la circulación de la información de manera equitativa a todos los miembros de una sociedad. Así, la labor periodística y de los medios de comunicación está implícita en los Derechos Humanos proclamados en diciembre de 1948, ya que como versa el Artículo 19 de dicho documento, el derecho a la comunicación es un derecho humano fundamental y los Estados deben garantizar la libertad de expresión y de creencias, además de garantizar la libertad de expresión pero, ante todo, el mismo artículo estipula el derecho a recibir información por cualquier medio, sin importar la frontera. Bajo estos preceptos, consideramos que los medios de comunicación y el periodismo tienen la obligación de generar un pensamiento ético, critico e informado con la finalidad de garantizar el derecho fundamental a la información.

El presente artículo profundiza sobre este tema exponiendo la importancia del pensamiento critico en los medios, el combate a las llamadas fake neww* ${ }^{*}$ una revisión de las garantías que se ofrecen en la actualidad en los medios a los Derechos Humanos en general.

Palabras clave: Derechos Humanos, Periodismo, Comunicación, Fake news, Pensamiento crítico.

\footnotetext{
*Tomamos este concepto de Javier Contreras, Premio Nacional de Periodismo 2019, quien utiliza el término para designar noticias falsas, que son esparcidas en la red, en particular en las redes sociales y que causan entre otras cosas desinformación y extravío de la realidad.
} 


\section{Abstract}

One of the essential tasks of journalism and communication is the circulation of information in an equitable manner to all members of a society. Thus, journalistic and media work is implicit in the Human Rights proclaimed in December 1948, since, as stated in Article 19 of said document, the right to communication is a fundamental human right and the States must guarantee the freedom of expression and belief, in addition to guaranteeing freedom of expression but, above all, the same article stipulates the right to receive information by any means, regardless of the border. Under these precepts, we consider that the media and journalism have the obligation to generate ethical, critical and informed thinking in order to guarantee the fundamental right to information.

This article delves into this subject by exposing the importance of critical thinking in the media, the fight against so-called fake news and a review of the guarantees currently offered in the media to $\mathrm{Hu}^{-}$ man Rights in general.

\section{Keywords: Virtual Reality, Freedom, Surveillance, Market.}

\section{RESUMO}

Uma das tarefas essenciais do jornalismo e da comunicação é a circulação de informações de maneira eqüitativa a todos os membros de uma sociedade. Assim, o trabalho jornalístico e midiático está implícito nos Direitos Humanos proclamados em dezembro de 1948, uma vez que, conforme consta do artigo 19 do referido documento, o direito à comunicação é um direito humano fundamental e os Estados devem garantir a liberdade de expressão e crença, em além de garantir a liberdade de expressão, mas, sobretudo, o mesmo artigo estipula o direito de receber informação por qualquer meio, independentemente da fronteira. Sob esses preceitos, consideramos que a mídia e o jornalismo têm a obrigação de gerar um pensamento ético, crítico e informado para garantir o direito fundamental à informação.Este artigo se aprofunda neste assunto ao expor a importância do pensamento crítico na mídia, o combate às chamadas notícias falsas e uma revisão das garantias atualmente oferecidas pela mídia aos Direitos Humanos em geral.

\section{Palavras-chave: Direitos Humanos, Jornalismo, Comunicação, Fake news, Pensamento crítico.}




\section{INTRODUCCIón}

La realidad es algo escurridizo, llegar a ella es tarea compleja, se requiere de un gran esfuerzo por parte del que quiere alcanzarla, una persona puede tener una serie de sesgos para llegar a la realidad. Primero que nada, están las prenociones del individuo, en donde se encuentran las creencias, valores y formación de la persona; segundo las sensaciones que despierta el objeto en el sujeto y por último luego de este proceso la persona evalúa y dará como resultado lo que será la realidad. Como es natural, en dicho proceso influyen las opiniones e influencias del entorno como puede ser la familia, la religión, la escuela y los medios de comunicación.

Cuando somos niños nuestra realidad en mucho es proveída por nuestros padres, maestros y demás figuras de autoridad, conforme vamos creciendo es natural que comencemos a formar nuestros propios criterios y consumos para configurar nuestra realidad. Es justo en este punto donde debemos de generar ejercicios de pensamiento crítico a nuestro entorno, cuestionar y contrastar lo que se nos presenta ante nuestros sentidos. En el presente trabajo entendemos el pensamiento crítico como Frida Díaz interpreta de McMillan:

El pensamiento crítico involucra el reconocimiento y comprensión de los supuestos subyacentes a lo que alguien afirma, la evaluación de sus argumentos y de las evidencias que ofrece, la realización de inferencias y la posibilidad de alterar los juicios realizados cuando sea justificado.

Por un lado, implica la posesión de conocimientos, la posibilidad de realizar una indagación lógica y razonar convenientemente, pero, por otra parte, también requiere de una actitud, del estar dispuesto a considerar los problemas de una manera perceptiva y reflexiva (Díaz 3)

Lo difícil del asunto es que, así como nosotros constantemente estamos formando nuestra realidad también los otros, esos con los que convivimos, están realizando la misma tarea y el resultado es que se crean complejos sistemas sociales; desafortunadamente dichos sistemas no siempre están enfocados al bienestar del sujeto, sino que sirven a intereses políticos, económicos y sociales que responden a intereses personales o de grupo.

Prueba de ello es que en la mayoría de los casos vivimos en una realidad en la que sabemos que comemos mal, no se cuenta con las mejores condiciones laborales y que las instituciones sociales, esas que deberían proveernos de bienestar; no están haciendo tan buena labor, así vivimos llenos de culpas y prohibiciones, somos informados por algunos medios de comunicación en la cultura del miedo, las llamadas fake neww, el constante estado de crisis y en muchos casos del terror. 
Toda esta confusión entre lo que es y lo que debería ser nace en la Modernidad, la cual entendemos en este texto como un proceso histórico a la manera que reseña Enrique Dussel en su texto "Europa, modernidad y eurocentrismo" en el que plantea que a partir de 1492 el mundo atravesó un proceso de mundialización en el que Europa era el centro y de ella emanaba todo la razón y bienestar, luego en el siglo XVIII señala el mismo Dussel, vinieron otros eventos como lo fue la Revolución Francesa, La Ilustración o la Revolución Industrial que abonaron a los conceptos de Europa conducida por la razón.

Lo más lamentable es que la historia nos ha mostrado que lejos de esta visión que nos vendió la Modernidad en la que nos esperaba un futuro prometedor, y hasta paradisiaco, estamos cada vez más inmersos en realidades de pobreza, desigualdad, inestabilidad política, depredación del medio ambiente y con terribles prácticas como la corrupción, el machismo, la violencia y la discriminación. ¿Pero cómo enderezar el rumbo? Al inicio de este texto se expresó que una de la solución es una visión crítica de la realidad, una visión de cuestionar, escudriñar a nuestras instituciones y, a la inversa, nuestras Instituciones, motivadas por el conjunto de opiniones críticas cuestionarse y replantearse. La escuela, la familia y los medios de comunicación, por mencionar solo algunos deben replantearse sus paradigmas, ir al fondo de sus motivaciones y preparar a los individuos para presentar una realidad que sea proactiva, con pensamiento de construcción de mejores condiciones para todos y todas.

Concretamente en el caso de los medios de comunicación, en este contexto de incertidumbre extrema, provocado por la Modernidad y la consecuente postmodernidad, diría Jurgen Lyotard (2004), ${ }^{1}$ estos poderosos vehículos de la realidad deben apelar a las máximas del periodismo y la comunicación que son las de informar, develar y generar procesos de pensamiento complejos y críticos en las audiencias. Ya no puede haber cabida para medios en pleno romance con el poder político, con grupos filosóficos o religiosos que solo busquen el beneficio sectario. Al respecto es conveniente mencionar que la Organización de las Naciones Unidas para la Educación, la Ciencia y la Cultura (unEsco), participa de la carta emanada de la Asamblea General de las Naciones Unidas en 1948 en lo relativo a la libertad de expresión del derecho a la información como un derecho humano y es por ello que como sociedad debemos apelar a la necesidad de exigir un contexto en el que la información emanada de los medios de comunicación, tradicionales como la radio, la televisión o la prensa escrita, como los nuevos, como lo son los medios electrónicos respeten un derecho humano que es el acceso a la información.

1 Lyotard es uno de los teóricos más reconocidos de la posmodernidad, apela por la caída de los grandes relatos y se decanta por la explicación de la realidad fragmentada. 
Los medios deben erradicar en lo inmediato la apología de la violencia, el machismo y el miedo colectivo y con ello nos referimos tanto con sus audiencias como con su personal. Es lamentable que en medio de la pandemia de COVID-19, que cimbró las vidas de todo el globo en este 2020 se continúen las prácticas de misoginia y de manejo tendencioso de la información.

Es labor de los comunicadores y periodistas generar dinámicas constructivas, ojo, no postulamos voltear la mirada ante lo modificables, sino el crear dinámicas de dar lugar a todos los actores, cuestionar a las fuentes y ver cada pequeño detalle detrás de cada contexto, y luego solo así presentar la información. Esto por supuesto que generará, y siguiendo con el hilo de configuración de lo real, escenarios integrales para que sean tomados por todos y todas.

\section{UN MUNDO MODERNO EN LA PRÁCTICA, PERO NOMINAL- MENTE POSMODERNO Y CON UNA VORAZ INMEDIATEZ EN LAS NOTICIAS}

Pandemias, desastres ambientales, un sistema capitalista poniendo constantemente a prueba la democracia y sus gobiernos y a los seres humanos de todo el mundo. Sistemas educativos, de salud, vivienda y acceso a los servicios básicos colapsados por la privatización propia del neoliberalismo. Todo muy lejos del discurso de la Modernidad en la que la humanidad pretendía llegar a un substancial avance, en donde los adelantos tecnológicos, el libre mercado abrirían terreno al bienestar, como sabemos el resultado es totalmente diferente, Enrique Dussel en sus conferencias "Dialéctica de la Modernidad" explica que Europa en su encuentro con el Nuevo Mundo de igualmente inauguró el pensamiento globalizante en el que el discurso de lo moderno se implanta, subordina a las civilizaciones de lo que a la postre se convertiría en América y apoyado con el sistema de producción capitalista cada vez más basado en dominaciones colonialistas desarrolla una dependencia a un gran numero de seres humanos a lo largo del mundo.

Posteriormente, destaca Dussel que Jurguen Habermas sitúa el inicio de la Modernidad en el siglo xvin apuntando que "los acontecimientos históricos claves para la implantación del principio de la subjetividad (moderna) son la Reforma, la Ilustración y la Revolución Francesa" (Habermas)

Iniciará en el siglo xviı como lo apuntó Habermas o con la llegada de los europeos al Nuevo Mundo como lo señala Dussel la realidad es que el discurso globalizado totalizante inunda el mundo y se construye una epistemología colonialista que nace desde Europa 
para el mundo. Hoy en día el discurso emerge en otras modalidades, aparentemente la Modernidad termina y vivimos, como lo señala Jean Francois Lyotard (2004) en la posmodernidad. Para este pensador, la posmodernidad se caracteriza por la finalización de los grandes discursos entre los que encuentra el marxismo y el cristianismo y se desenvuelven una multiplicidad de interpretaciones de la realidad donde todo es relativo. Pero esto no es privativo de la presencia de la Modernidad, en la práctica el discurso del progreso que vende lo Moderno continua presente, ese discurso en el que todo se puede explicar, aunque en la realidad todo sea relativo.

Se realizaron estas precisiones a propósito de que por un lado existe el gran consenso a partir de mediados del siglo xx de que existe una carta de los Derechos Humanos que aplica a todos los pueblos del globo, en la que organizaciones como la UNEsco protegen los derechos a la educación, la cultura y la información y por el otro los relativismos están a la vista de todos y todas. Los científicos del mundo, en especial los de las ciencias sociales desde la entrada del posmodernismo han estudiado fenómenos, como el educativo, en el que las nuevas interpretaciones nacen con fuerzas inusitada y luchan contra el poder totalizante de la educación tradicional.

En el periodismo ocurre algo similar, por un lado emanado de manuales de ética y cimentados en Derechos Humanos que garantizan el acceso a la información como una manera de democratizar el acceso a lo ocurrido en el planeta y ante todo como una herramienta de toma de consciencia contra los mensajes de odio que pudieran valerse de los medios de comunicación de masas y perseguir intereses particulares o sectarios.

La Declaración sobre los Principios Fundamentales relativos a la Contribución de los Medios de Comunicación de Masas al Fortalecimiento de la Paz y la Comprensión Internacional, a la Promoción de los Derechos Humanos y a la Lucha contra el Racismo, el Apartheid y la Incitación a la Guerra es un documento de la UNEsco promulgado el 28 de noviembre de 1978. En el que se contempló por medio de la Conferencia General que:

la UNEsco se propone "contribuir a la paz y a la seguridad estrechando, mediante la educación, la ciencia y la cultura, la colaboración entre las naciones a fin de asegurar el respeto universal a la justicia, a la ley, a los derechos humanos y a las libertades fundamentales" (art. 1,1), y que para realizar tal finalidad la Organización se preocupará de "facilitar la libre circulación de las ideas, por medio de la palabra y de la imagen” (art. 1, 2). (Unesco1978)

En este mismo sentido, y reunidos en la misma asamblea se plantea la necesidad de que en virtud de la Declaración universal de los Derechos Humanos aprobada en 1948 en el artículo 19 se estipula: 
todo individuo tiene derecho a la libertad de opinión y de expresión; este derecho incluye el de no ser molestado a causa de sus opiniones, el de investigar y recibir informaciones y opiniones, y el de difundirlas, sin limitación de fronteras, por cualquier medio de expresión”, así como el Pacto Internacional de Derechos Civiles y Políticos, aprobado por la Asamblea General de las Naciones Unidas en 1966, que proclama los mismos principios en su artículo 19 y que en su artículo 20 condena la incitación a la guerra, la apología del odio nacional, racial o religioso así como toda forma de discriminación, de hostilidad o de violencia. (unEsco 1978).

De la misma manera como un antecedente de la declaración de 1978 se estableció en la Asamblea General de las Naciones Unidad desde 1946 que:

La libertad de información es un derecho humano fundamental y piedra de toque de todas las libertades a las cuales están consagradas las Naciones Unidas; (...) La libertad de información requiere, como elemento indispensable, la voluntad y la capacidad de usar y no abusar de sus privilegios. Requiere, además, como disciplina básica, la obligación moral de investigar los hechos sin prejuicio y difundir las informaciones sin intención maliciosa... (unesco 1978).

Esos fueron los antecedentes que le dan cuerpo a la declaración de 1978 en la que se enuncia como esencia el derecho a la información y al libre ejercicio del periodismo. Es una carta que no tiene desperdicio y que a continuación enunciaremos algunos de sus postulados más significativos en lo relativo al acceso a la información y el ejercicio del periodismo, el articulo in establece en sus tres primeros apartados:

1. El ejercicio de la libertad de opinión, de la libertad de expresión y de la libertad de información, reconocido como parte integrante de los derechos humanos y de las libertades fundamentales, constituye un factor esencial del fortalecimiento de la paz y de la comprensión internacional.

2. El acceso del público a la información debe garantizarse mediante la diversidad de las fuentes y de los medios de información de que disponga, permitiendo así a cada persona verificar la exactitud de los hechos y fundar objetivamente su opinión sobre los acontecimientos. Para ese fin, los periodistas deben tener la libertad de informar y las mayores facilidades posibles de acceso a la información. Igualmente, los medios de comunicación deben responder a las preocupaciones de los pueblos y de los individuos, favoreciendo así la participación del público en la elaboración de la información.

3. Con miras al fortalecimiento de la paz y de la comprensión internacional, de la promoción de los derechos humanos y de la lucha contra el racismo, el apartheid y la incitación a la guerra, los órganos de información, en todo el mundo, dada la función que les corresponde, contribuyen a promover los derechos humanos, en particular haciendo 
oír la voz de los pueblos oprimidos que luchan contra el colonialismo, el neocolonialismo, la ocupación extranjera y todas las formas de discriminación racial y de opresión y que no pueden expresarse en su propio territorio. (UNEsco 1978)

Una vez revisado los aspectos del contexto de la modernidad y como a la par nace la proclamación de los derechos humanos podemos adentrarnos al estudio del cómo está responsabilidad reviste el oficio del periodismo y la comunicación. Ha quedado claro que son derechos humanos que propician la igualdad entre los pueblos y previenen la violencia y hacen más corta la separación entre las personas, pero aquí es conveniente el cuestionarse si un solo discurso que es el de los Derechos Humanos en la realidad se aplica en el periodismo y la comunicación en nuestros días, y sobre todo si en un contexto global con la entrada en desde finales del siglo pasado del internet y su consecuente conectividad global y la circulación de la información permiten el co-

102 rrecto respeto al derecho humano del acceso a la información.

Para saber si esto se logra es necesario valerse de uno de los tópicos más utilizados en los últimos tiempos, las llamadas fake news o noticias falsas que en parte son provocadas porque los medios de comunicación se ven forzados a la inmediatez y circulan información en muchas ocasiones falsa, y su vez esto cae en dos paradigmas altamente presentes en la sociedad actual, uno es la desinformación y otra es la infodemia ${ }^{2}$ o saturación de noticias.

Si para analizar lo anterior nos vamos a los porque de los medios de comunicación dentro del periodismo y la comunicación existen las máximas de brindar información veraz, el cruce de fuentes y presentar a las audiencias una versión sin balances para uno de los extremos de las noticias. Ese es el discurso propio de la profesión del informar, digamos el gran discurso, pero si en la práctica en el mundo actual se presentan fenómenos desencadenados por la inmediatez que brinda la conectividad y el medio de comunicación que de primero la noticia es el que se lleva la audiencia, muchos medios, tanto tradicionales como los nuevos electrónicos faltan a las máximas antes mencionadas y caen en las llamadas fake news que se amontonan en los ordenadores y teléfonos inteligentes de las personas, en las radios, los periódicos y las televisiones causando como ultimo eslabón desconcierto.

En palabras de Cebrián (2012), citada por Judith Herrera (2020) "en un mundo cada vez más convulsionado, la instantaneidad

2 La Infodemia es un concepto que se ha utilizado en los tiempos del COVID-19 para llamar a la saturación de información que sufren las personas por medio de los canales de información tradicional como lo es la radio, la televisión, el periódico, pero también vía las redes sociales $\mathrm{t}$ medios electrónicos. 
se ha vuelto la norma en los medios de comunicación; se debe informar sobre un hecho con la mayor inmediatez posible. No se trata de una realidad aislada, sino que responde a la evolución de las tecnologías de conectividad y también al auge de las redes sociales”. Esto refuerza lo que se había comentado anteriormente el periodista se encuentra cada vez más expuesto por la presión de su profesión a brindar una información cada vez de manera más rápida lo que hace que muchos portales y medios falten a su vez a las máximas éticas de la comunicación y el periodismo y contribuyan a la desinformación y por ende lo que se mencionó al inicio de este escrito, una configuración desfigurada de la realidad, la misma Herrera (2020), cita a Miquel Rodrigo Alsina (2005) en el que habla de la construcción que el periodista realiza de la realidad:

Dentro de la perspectiva de la construcción social de la realidad, concibo la construcción de la noticia como un especial de realidad: es la realidad simbólica, pública y cotidiana (...) los periodistas son, como todas las personas, constructores de la realidad en su entorno. Pero además, dan forma de narración a esta realidad y, difundiéndola, la convierten en una realidad pública sobre el acontecer diario (Alsina citado en Herrera 13).

Con esa enorme responsabilidad y desventaja se ejerce el periodismo, siguiendo el mismo objetivo que ya marca Rosentiel y Kovach (2003) "el propósito principal del periodismo es proporcionar a los ciudadanos la información que necesitan para ser libres y capaces de gobernarse a sí mismos".

Con el tema relativo a las noticias falsas estas abundan en los medios y ocurren en todos los niveles sin que necesariamente se desmienta el suceso, pero es labor del periodista el verificar siempre las noticias y como menciona Herrera (2020) citando Eva Herrero cuando menciona que:

El periodista debe ser capaz de filtrar, comprobar y acreditar esos nuevos contenidos que se generan en los social media y llevar a cabo un trabajo informativo completo y eficaz, sin olvidar la veracidad y el rigor que debe desprenderse de cualquier noticia (Herrero 3 ).

Desgraciadamente menciona Herrera (2020) la constatación de las informaciones no siempre se pueden realizar y presentan diferentes dificultades en las mesas de redacción, por ejemplo la noticia que se difundió a principios del siglo en la que se aseguraba que Irak contaba con armas de destrucción masiva requería de una verificación con altos mandos del ejercito norteamericano y si le agregamos que de ese momento a la fecha las redes sociales producen grandes cantidades de información en muchas ocasiones las noticias falsas son incontenibles y desencadenan una confusión generalizada en lo social. 


\section{LAS CONCLUSIONES}

Luego de realizar una revisión que pasó desde el nacimiento de la Modernidad, la llegada de la Posmodernidad, la Declaración de los derechos Humanos, la participación de la unesco en la garantía del derecho a la información y terminar con un panorama del escenario del ejercicio del periodismo y la comunicación y su lucha contra las noticias falsas, todo en aras de defender las máximas de la profesión, debemos de apelar al concepto que se planteó al inicio de este articulo, el pensamiento crítico es la respuesta. El pensamiento crítico permite a las personas ser consientes de su realidad, realizar un contraste de la información que se le brinda y sacar resultados de los mismos. Pero, ¿de dónde sacamos pensamientos críticos en nuestros días? Como se evidenció, por lo menos en grandes sectores del ejercicio del periodismo, no se está realizando un periodismo que respete de entrada los Derechos Humanos, por el contrario, se está contribuyendo a la desinformación, en casos a la división social y a convertir a los medios en portadores de noticias falsas por los canales tradicionales y los nuevos. Por todo ello es primordial que como se ha intentado en este trabajo se propongan propuestas de acción, propuestas políticas que sin entrar en la limitación del derecho de expresión si profundicen en la generación del pensamiento crítico en aquellos que practican el periodismo y la comunicación. Esta es una labor de gobiernos, pero también de autoridades educativas generando pedagogías que propicien el pensamiento crítico y complejo en las personas. Es labor de los medios de comunicación ya que como se evidenció deben responder a normas internacionales, como lo son los Derechos Humanos, pero también de legislaciones nacionales. Es labor de los y las periodistas y comunicadores que deben luchar por su derecho al acceso a la información y desprenderse de las cúpulas de poder tanto locales, como nacionales e internacionales que dan espacios a discursos de odio y terror. En general es una acción de todos y todas.

Es verdad que la Modernidad, el capitalismo venden escenarios de éxito y que estos se han tornado apocalípticos, que la posmodernidad es al extremo relativa, pero al final lo que importa es que desde una postura política y critica se puede robustecer el ejercicio del informar, aprovechar el auge que tienen los nuevos discursos, dar la voz en los medios a niños y niñas, personas desplazadas, mujeres que sufren de violencia, personas bajo el racismo, migrantes, comunidades indígenas y en general toda aquella persona que desee poner el dedo en yaga a las problemáticas mencionadas u otras que nos unen como la destrucción del medio ambiente y la voracidad del ser humano en esta lucha por el individualismo. 
A aparte de todo lo mencionado nace una disyuntiva muy importante, Immanuel Wallerstein (2015) en su libro sobre Universalismo europeo habla sobre los discursos que han creado una subordinación globalizante en el mundo, comenzando con el de la conquista de América en el que se subordinó a los habitantes del nuevo mundo so pretexto de la evangelización, luego este mismo autor recrea la manera en que las potencias europeas se extendieron a lo largo del mundo "no civilizado" en el siglo xIx llevando los adelantos a extensos territorios como el continente africano y Asía. Explica que para mediados del siglo xx este discurso fue imposible continuar sosteniéndolo por lo que la Declaración de los Derechos Humanos fue la razón siguiente para subordinar a los pueblos. Wallerstein (2015) explica que con la creación y difusión de los Derechos Humanos y los centinelas mundiales que son las ONGs hoy se señala a diestra y siniestra los errores en los países y que es el perfecto pie para intervencionismo del llamado primer mundo. En lo personal me parece sumamente preocupante y que es susceptible de posibles futuros estudios ya que significaría un nuevo gran discurso totalizante de creación de desigualdades entre dominantes y dominados.

\section{REFERENCIAS}

Díaz, Frida. "Habilidades de pensamiento crítico sobre contenidos históricos en alumnos de bachillerato." Revisa Mexicana de Investigación Educativa Sep. (2001), 6-13. Web 20 oct 2020.

Dussel, Enrique. Europa, modernidad y eurocentrismo. Universidad Autónoma Metropolitana (UAM-I), (2000), CLASCO México, 2000, Print.

—. (14 agosto 2016) "Dialéctica de la modernidad,origen, desarrollo y ocaso. Clase 1” recuperado de: https://www.youtube.com/watch?v=kC$\mathrm{d} 3 \mathrm{DJcPtqE} \& \mathrm{t}=4 \mathrm{~s}$

Habermas, Jurgen. Der philosophische Diskurs der Moderne, Suhrkamp. Ed en español: El discurso filosófico de la modernidad. Taurus Humanidades, España. 1989, Print.

Herrera, Judith. "Verificación de noticias y el problema de la inmediatez: análisis de cobertura de una noticia falsa en medios digitales chilenos.” Anuario Electrónico de Estudios en Comunicación Social. Disertaciones Ene. (2020). Web 20 oct 2020.

Herrero, E, "El periodismo en el siglo de las redes sociales." Revista de Comunicación Dic (2011), 117. Web 20 oct 2020. 
LAS FAKE NEWS, ENEMIGAS DEL PERIODISMO

Kovach, B. Rosentiel. Los elementos del periodismo. Aguilar, España. 2003.

Lyotard, Jean. La Condición posmoderna, Informe del saber. Colección Teorema, Serie Mayor. Catedra, España. 2004.

unesco. Declaración sobre los Principios Fundamentales relativos a la Contribución de los Medios de Comunicación de Masas al Fortalecimiento de la Paz y la Comprensión Internacional, a la Promoción de los Derechos Humanos y a la Lucha contra el Racismo, el Apartheid y la Incitación a la Guerra. Sep (1978) Web. Oct. 2020.

Wallerstein, Immanuel. Universalismo europeo, el discurso del poder. Ed. Siglo. XXI, México, (2015). 